Methods This was a retrospective analysis of patients who underwent ESD or hybrid ESD/endoscopic piecemeal resection (ESD/ EPMR) of large benign rectal polyps. Details pertaining to the polypectomy were gathered and patients were asked a series of questions regarding their bowel function before and after endoscopic resection.

Results Thirty four patients underwent ESD or hybrid ESD/EPMR for large rectal polyps between January 2006 to May 2012. The median maximal polyp measurement was $62 \mathrm{~mm}$ (range 40 $150 \mathrm{~mm}$ ) and median circumferential involvement, $50 \%$ (range $30-$ $90 \%$ ). The patients had symptoms of the passage of mucous (12 patients), rectal bleeding (9)and faecal incontinence (1) before the treatment. In all cases, these symptoms resolved after the treatment. Regarding the bowel function post procedure, 16 patients (47.1\%) opened their bowel less frequently, 12 patients (35.3\%) the same and 6 patients $(17.6 \%)$ more frequently. Eighteen patients felt that their completeness of evacuation was the same, 8 improved and 5 worsen. Almost all of the patients (33 patients, 97.1\%) did not use laxatives before their polypectomy, however 14 patients $(41.2 \%)$ required the regular use of laxatives post procedure. None of the cases did not require any further intervention like dilatation. There was no relationship between circumferential involvement and patients who developed a decreased frequency of defecation or who used laxatives after ESD (Mann-Whitney Test, $p=0.567$ ). On a scale of 1 to 10 , where 1 was extremely unsatisfied and 10 was extremely satisfied, the patients rated their ESD experience at 10 (Median, Range 7 - 10).

Conclusion ESD of large rectal polyps resulted in an improvement in symptoms of rectal bleeding and passage of mucous. It also results in a decreased frequency of defecation and an increase in use of laxatives. Functional outcomes are not related to polyp size or circumferential involvement. Patients were extremely satisfied with their ESD experience.

Disclosure of Interest None Declared.

\section{PTH-033 A SYSTEMATIC APPROACH TO STANDARD ENDOSCOPIC SAMPLING OF BILE DUCT STRICTURES IS HIGHLY ACCURATE IN THE DIAGNOSIS OF BILIARY STRICTURES}

doi:10.1136/gutjnl-2013-304907.520

1,*0 Noorullah, ' V P K Lekharaju, ' $\mathrm{C}$ W Wadsworth, ${ }^{2} \mathrm{~K}$ Brougham, ${ }^{1} \mathrm{~N}$ Stern, ${ }^{1} \mathrm{~S}$ Hood, ${ }^{1} \mathrm{C}$ Kaltsidis, ${ }^{2} \mathrm{M}$ Terlizzo, ${ }^{1} \mathrm{R}$ Sturgess. 'Digestive Diseases Unit; ${ }^{2}$ Department of Pathology, Aintree University Hospital, Liverpool, UK

Introduction Accurate diagnosis of bile duct strictures as malignant or benign is imperative for optimal patient management, but is frequently difficult. Histological and cytological samples can be obtained at ERCP. Various techniques have been studied and reported specificities are generally very high. Sensitivities are modest and variable. The reported sensitivity is $33-57 \%$ for brush cytology and $43-81 \%$ for transpapillary histology. The yield may be increased by combining two sampling methods.

Methods Data was collected prospectively on all patients with bile duct strictures who underwent histology and cytology. Transpapillary, intraductal biopsies were obtained using a paediatric biopsy forceps (Boston Scientific, Radial Jaw 4, 2.0mm paediatric biopsy forceps, Hemel Hempstead, UK). Fluoroscopic guidance was used to selectively target the stricture. A minimum of 4 biopsies were obtained. Cytological samples were obtained using an over the wire brush (Boston Scientific, RX Biliary brush $2.1 \mathrm{~mm}$, Hemel Hempstead, UK). This involved multiple passes through the stricture and withdrawal of the brush in to the catheter after the final pass. On retrieval, the catheter was flushed with the cytology fixation fluid in to the cytology collection container and the brush was cut off and sent in the same container.
The sample was delivered to the lab for processing to the lab immediately after the procedure. Patients diagnosed with benign strictures had a follow up with median length of 20 months (range 4-35).

Results 96 strictures were sampled using dual modality. 70 were malignant of which 49 were in the peri-hilar and proximal CBD and 21 in distal CBD. The sensitivity in diagnosis of malignant biliary strictures was $74 \%$ with an overall accuracy for all strictures of $81 \%$

Histology was positive in $33 / 52$ cases (63\%) and cytology was positive in $29 / 52$ cases (56\%). Taken together, they yielded a significantly better result of $74 \%$.

The sensitivity was higher for peri-hilar (82\%) as compared to distal strictures $(57 \%)$. There were no false positives. No complications as direct result of either tissue acquisition techniques occurred.

\begin{tabular}{|c|c|}
\hline $\begin{array}{l}\text { Sensitivity } \\
\text { Peri-hilar } \\
\text { Distal }\end{array}$ & $\begin{array}{l}74 \%(n=70) \\
82 \%(n=49) \\
57 \%(n=21)\end{array}$ \\
\hline Specificity & $100 \%$ \\
\hline PPV & $100 \%$ \\
\hline NPV & $59 \%$ \\
\hline Accuracy & $81 \%$ \\
\hline
\end{tabular}

Conclusion A highly systematic approach and combination of histology and cytology offers a significant advantage in diagnostic accuracy for both malignant and benign strictures. The high yield on cytology in our study may reflect attention to detail and prompt processing in the lab. None of the techniques adopted required specialised equipment or skills and could be adopted by any ERCP unit

Disclosure of Interest $\mathrm{O}$. Noorullah: None Declared, V. Lekharaju: None Declared, C. W. Wadsworth: None Declared, K. Brougham: None Declared, N. Stern: None Declared, S. Hood: None Declared, C. Kaltsidis: None Declared, M. Terlizzo: None Declared, R. Sturgess Conflict with: Advisory board member and in receipt of honoraria from Olympus UK and Boston Scientific

\section{PTH-034 ACHIEVING NICE STANDARDS FOR ENDOSCOPIC SUBMUCOSAL DISSECTION: FIRST UK PRACTICE STUDY}

doi:10.1136/gutjnl-2013-304907.521

1,*P Hurlstone, 'I Elfadel, 'E Said. 'Endoscopy, Barnsley NHS Foundation Trust, Yorkshire, UK, Barnsley, UK

Introduction Endoscopic submucosal dissection (ESD) is an advanced technique that aims to resect appropriately selected gastrointestinal intraepithelial neoplasias en bloc, allowing precise histological diagnosis for staging and resection margin analysis. No published data from the UK exists from either tertiary referral units or DGH settings since the recent publication of ESD NICE guidelines in 2010. Such data is of paramount importance for service development and for establishing the potential role of ESD in current Cancer Service Frameworks (CSF) in the UK. Data is urgently required by NICE for stage 1 guidance revision.

Methods All patients who underwent ESD in 2009-2012 at BDGH $(\mathrm{n}=47)$ were included in this study. Primary outcome measures were complication rates, resection status, 30-day readmission rates, mortality and tumour recurrence. These data were then analysed against recently published standards set by NICE.

Results Inclusions-HGD/IMC/T1 carcinoma/GIST. $\mathrm{n}=47$ (LGIT 26/UGIT 21). En bloc resection was achieved in $45 / 47$ 